\title{
ANÁLISIS DE LOS ASPECTOS ÉTICOS DE LA INVESTIGACIÓN EN SERES HUMANOS CONTENIDOS EN LAS INSTRUCCIONES A LOS AUTORES DE 139 REVISTAS CIENTÍFICAS BRASILEÑAS
}

\author{
Trajano Sardenberg, \\ Sergio S. Müller, HamiltonR. Pereira, \\ Reinaldo A. Oliveira, William S. Hossne \\ Institución: Departamento de Cirurgia e Ortopedia, \\ Faculdade de Medicina de Botucatu, \\ Universidade Estadual Paulista "Júlio de Mesquita Filho" - UNESP, \\ Botucatu, São Paulo, Brasil.
}

Correspondencia: Dr. Trajano Sardenberg, Departamento de Cirurgia e Ortopedia, Faculdade de Medicina de Botucatu, Universidade Estadual Paulista "Júlio de Mesquita Filho” - UNESP, Botucatu, São Paulo, Brasil. - Cep18.618-970 - Fax: (02114) 821-7615 E-mail: tsarden@fmb.unesp.br 



\title{
ANÁLISIS DE LOS ASPECTOS ÉTICOS DE LA INVESTIGACIÓN EN SERES HUMANOS CONTENIDOS EN LAS INSTRUCCIONES A LOS AUTORES DE 139 REVISTAS CIENTÍFICAS BRASILEÑAS
}

\author{
Trajano Sardenberg, \\ Sergio S. Müller, HamiltonR. Pereira, \\ Reinaldo A. Oliveira, William S. Hossne
}

\section{Resumen}

La preocupación sobre la ética de la investigación en seres humanos ha crecido en Brasil durante la última década. El análisis de los aspectos éticos contenidos en las "Instrucciones a los Autores" de las Revistas Científicas Brasileñas puede contribuir a la comprensión de la política editorial científica brasileña.

Palabras clave: Ética de la investigación, Control ético de la investigación, Código, Ética de la comunicación, Ética de la educación.

\begin{abstract}
The concern with ethical standards during research in human subjects has been improving in Brazil during the last decade. Analysis of the ethical recommendations in instructions to authors may contribute to understand Brazilian Scientific Journals policies.
\end{abstract}

Key words: Research ethics, Ethical control in research, Code, Ethics of communication, Ethics of education.

\section{Resumo}

A preocupação com a ética na pesquisa em seres humanos tem crescido no Brasil durante a última década. A análise dos aspectos éticos contidos nas Instruções aos Autores das revistas científicas brasileiras pode contribuir para a compreensão da política editorial científica brasileira.

Palavras chaves: Ética da pesquisa, Control ético da pesquisa, Código, Ética da comunicação, Ética da educação.

\section{Résumé}

La préocupation concernant l'éthique de la recherche sur des êtres humains a augmenté au Brésil pendant la dernière décennie. L'analyse des aspects éthiques contenus dans les "Instructions aux Auteurs" des Revues Scientifiques Brésiliennes peut contribuer à la compréhension de la politique d'édition scientifique brésilienne.

Mots clés: Éthique de la recherche, Contrôle éthique de la recherche, Codes, Éthique de la communication, Éthique de l'éducation. 



\section{Introducción}

El Tribunal de Nüremberg, que juzgó los Crímenes de Guerra de la Segunda Guerra Mundial en 1947, elaboró el Código de Nüremberg (1), que establece 10 normas que los médicos deben respetar cuando desarrollan experimentaciones con seres humanos. La primera norma, la más importante y detallada, establece que el "Consentimiento voluntario de los sujetos humanos es absolutamente necesario". No existen referencias de que los protocolos de investigación en seres humanos deban ser previamente aprobados por una comisión independiente, ni tampoco referencias relativas a la publicación de los resultados de estos estudios.

La Declaración de Helsinki de la Asociación Médica Mundial, de 1964 (revisada diversas veces, siendo la última edición aprobada en la $48^{\mathrm{a}}$ Asamblea General de la República de Africa del Sur en 1996) (2), afirma que los protocolos de investigación en seres humanos deben ser analizados por un comité independiente del investigador y que "los informes sobre investigaciones que no se ciñan a los principios descritos en esta declaración no deben ser aceptados para su publicación".

El Código de Ética Médica Brasileño (3) de 1988, contiene capítulos referentes a la investigación médica y al trabajo científico. Respecto de la investigación médica (nueve artículos), se refiere al consentimiento y a la necesidad de que los protocolos de investigación sean sometidos a la aprobación y acompañamiento de una comisión independiente del investigador. En el capítulo sobre el trabajo científico, no se mencionan aspectos éticos respecto de la publicación de los resultados de estudios en seres humanos. El Código de Ética de los Profesionales de Enfermería (4) y el Código de Ética Odontológica (5) especifican la necesidad del consentimiento informado para la realización de investigaciones en seres humanos.
El Consejo Nacional de Salud del Ministerio de la Salud (CNS-MS) editó en1988 (6) la Resolución no 1/88 que, entre diversos capítulos, establece la necesidad del "consentimiento post información" y exige que los protocolos de investigación sean aprobados por un Comité de Ética independiente del investigador, sin referirse tampoco a los aspectos éticos relacionados con la publicación de los resultados de la investigación en seres humanos. En 1996, el CNS-MS aprobó la Resolución 196/96 (7), que incorpora varios conceptos de la bioética y mantiene el consentimiento del individuo y la necesidad de un previo examen por el Comité de Ética. La Resolución 196/96 no estipula reglas en este sentido; no obstante, establece que los resultados de la investigación en seres humanos deben ser publicados, sean ellos favorables o no.

La preocupación sobre los aspectos éticos de la investigación en seres humanos en Brasil, a pesar de todos los documentos donde se hacen referencias oficiales para los investigadores, principalmente en relación con la aprobación por comisiones o comités de ética, fue particularmente impactada con la exigencia de diversas revistas científicas internacionales, especialmente las de lengua inglesa, de aceptar solamente, para análisis y posible publicación, los estudios cuyos protocolos hayan sido aprobados previamente por comisiones institucionales.

La publicación o no de artículos considerados inadecuados éticamente es parte de una antigua polémica en el medio científico y, no obstante, permanece extremadamente actual (8-13). Entretanto se produjo un nítido cambio en el enfoque, en tanto no se debate más sobre la publicación o no de artículos clara y genéricamente considerados no éticos, pero sí, si las revistas científicas deben publicar investigaciones que no incluyen el consentimiento informado en su ejecución, y no fueran analizadas y aprobadas por comités de ética institucionales. 
Análisis de los aspectos éticos de la investigación en seres humanos contenidos en las Instrucciones a los Autores de 139 revistas científicas brasileñas - T. Sardenberg y cols.

En las dos últimas décadas ha habido, entre los editores de revistas científicas internacionales, una constante preocupación por establecer orientaciones estandarizadas para la elaboración de manuscritos que deben ser sometidos a publicación, siendo fruto de este esfuerzo el Comité Internacional de Editores de Revistas Médicas, el cual edita los Requisitos Uniformes para Manuscritos Sometidos a Revistas Biomédicas (RUMSRB), actualmente seguido por más de 500 revistas, incluso en Brasil (14-16). Las orientaciones de los RUMSRB hacen referencia explícita a la Declaración de Helsinki y a comités responsables para la experimentación humana (regional o institucional). Más aún, se solicita a las revistas que concuerdan con los RUMSRB, que el documento sea citado en sus Instrucciones a los Autores.

Actualmente, gran parte de las revistas científicas del área de medicina y biomedicina de lengua inglesa hacen referencia a aspectos éticos de la investigación en seres humanos en sus Instrucciones a los Autores (17). No fue posible encontrar, en la literatura científica brasileña, publicaciones que estudiasen el impacto y la presencia de los paradigmas actuales de la bioética en la práctica editorial.

Delante de este cuadro decidimos analizar, a partir de las Instrucciones a los Autores de revistas científicas brasileñas de las áreas de medicina, biomedicina, enfermería, odontología y ciencias generales, los aspectos éticos referentes a las investigaciones en seres humanos.

\section{Material y métodos}

Las secciones de las Instrucciones a los Autores de 139 revistas científicas brasileñas de medicina, biomedicina, enfermería, odontología y ciencias generales fueron analizadas en relación con las orientaciones éticas de las investigaciones en seres humanos, de manera semejante al estudio de
Amdur y Biddle (17). Las revistas fueron agrupadas, cuando fue posible, de acuerdo con las especialidades médicas. Las revistas de Enfermedades Infecciosas, por ejemplo, fueron clasificadas en Clínica Médica y las de Ginecología y Obstetricia en el del área de Cirugía. Revistas sin áreas específicas fueron catalogadas en Medicina General y las publicaciones de Biomedicina, Biología y ciencias generales fueron agrupadas en Miscelánea. Todas las revista de Odontología fueron incluidas en el mismo grupo.

En relación a los aspectos éticos de las orientaciones contenidas en las Instrucciones a los Autores, principalmente en la parte relacionada a la investigación en seres humanos, las revistas fueron clasificadas en seis categorías:

Comisión o Comité de Ética: Revistas que hacen referencia a la necesidad de aprobación y/o análisis de los trabajos por Comisión de Ética de la institución donde el estudio fue realizado, independiente de haber otras recomendaciones. Por ejemplo, la revista del Colégio Brasileño de Cirujanos, clasificada en este grupo, señala que:

Toda materia relacionada con la investigación humana y la investigación animal debe tener la aprobación previa de la Comisión de Ética de la institución donde el trabajo fue realizado, de acuerdo con las recomendaciones de la Resolución de Helsinki y las Normas Internacionales de Protección a los Animales.

Declaración de Helsinki: Revistas en la cual no hay referencia a Comisión o Comité de Ética de la institución donde el estudio fue realizado, pero citan la Declaración de Helsinki, independiente de referencias a otros aspectos éticos. La revista del Hospital de Clínicas de Porto Alegre, por ejemplo, clasificada en este grupo, cita, en sus Instrucciones a los Autores, además de la Declaración de Helsinki, la resolución 
196/96 del Consejo Nacional de Salud y las resoluciones normativas sobre la investigación del Hospital de Clínicas de Porto Alegre.

Consentimiento del paciente: Revistas en que la única referencia a los aspectos éticos de la investigación en seres humanos es la solicitud de obtención del consentimiento del paciente.

Principios/Normas/Estándares Éticos: Revistas que hacen referencias genérica respecto de principios, normas, estándares y etc. éticos. Por ejemplo, la Revista Brasileña de Reumatología afirma "Los estándares científicos y éticos son básicos para la aceptación de cualquier tipo de trabajo".

Requisitos Uniformes: Revistas en las que hay referencia directa o indirecta a las orientaciones de los Requisitos Uniformes para Manuscritos Sometidos a Revistas Biomédicas, propuestas por el Comité Internacional de Editores de Revistas Médicas. No fueron clasificadas en este grupo las revistas que hacen referencia a lo RUMSRB solamente en relación a la presentación de las referencias bibliográficas y otros aspectos técnicos.

Sin orientación ética: Revistas que no hacen referencia a los aspectos éticos relacionados con la investigación en seres humanos. Revistas que hacen referencia solamente a los aspectos relacionados con la privacidad de los pacientes o que citan el término "paciente o casuística" en lugar de "material" también fueron clasificadas en este grupo.

La manera como los aspectos éticos de los artículos enviados para las revistas científicas deben ser informadas a los editores fueron clasificadas en cinco grupos:

Incluido en el texto del artículo: Revistas que indican claramente que las informaciones sobre los aspectos éticos de la investigación en seres humanos deben ser citados en el texto del artículo.
Carta firmada: Revistas que exigen carta o documento firmado por el autor informando los aspectos éticos del estudio.

Copia de la autorización del Comité o Comisión de Ética: Revistas que explicitan la exigencia del envío de copia de la autorización de la Comisión de Ética de la institución donde el estudio fue realizado, junto con el manuscrito de la investigación.

Subentendido: Revistas que informan, en las Instrucciones a los Autores, que el editor subentiende que el estudio fue realizado dentro de las normas, estándares o principios éticos, sin la exigencia de cualquier otro tipo de información.

Sin referencias: Revistas que no contienen ninguna información de como los autores de los manuscritos enviados para la publicación deben informar sobre los aspectos éticos del estudio.

\section{Resultados}

Los resultados del análisis de las orientaciones éticas solicitadas en las Instrucciones a los Autores de 139 revistas científicas brasileñas del área de medicina, biomedicina, enfermería, odontología y ciencias generales están resumidos en las tablas I, II, III y IV y cuadros 1, 2, 3 .

El número de revistas consideradas esencialmente médicas (clasificadas en cirugía, clínica médica, pediatría, medicina general, medicina social y ocupacional, oncología y especialidades) es de 79, de las cuales $24(30,3 \%)$ presentan alguna referencia a la ética de investigación en seres humanos; en el área de odontología, de un total de 44 revistas, solamente $3(6,8 \%)$ presentan orientaciones éticas.

Debido a la gran variedad en las referencias a los aspectos éticos de la investigación en seres humanos de las 
Análisis de los aspectos éticos de la investigación en seres humanos contenidos en las Instrucciones a los Autores de 139 revistas científicas brasileñas - T. Sardenberg y cols.

Instrucciones a los Autores, observado en las revistas estudiadas, se privilegió, en la clasificación adoptada, las relacionadas con las Comisiones o Comités de Ética.

Dentro de este contexto, varias revistas que hacen referencia a la Declaración de Helsinki, Código de Nüremberg y al Consentimiento esclarecido del paciente, fueron clasificadas en el grupo "Comité/ Comisión de Ética". No obstante, debido a la importancia de estos aspectos éticos, todas sus citas fueron evaluadas, constatando que la Declaración de Helsinki es citada seis veces, el Código de Nüremberg uno y el "Consentimiento del paciente", ocho veces.

\section{Discusión}

El presente estudio se limitó al análisis de las Instrucciones a los Autores, no siendo realizadas entrevistas con los editores, ni análisis de artículos publicados en estas revistas. Se trata, por lo tanto, de un análisis parcial de la política editorial en relación a la ética de estos periódicos. Entretanto, las orientaciones contenidas en la sección de Instrucciones a los Autores de las revistas es la primera $\mathrm{y}$, muchas veces, la única vía de comunicación entre investigadores y editor $\mathrm{y}$, por lo tanto, la política adoptada por los periódicos, reflejada en las Instrucciones a los Autores, adquiere una enorme importancia en la práctica de publicación de artículos científicos.

Goldemberg et al (18), analizando diversos aspectos de las Instrucciones a los Autores de 19 periódicos médicos brasileños, subrayan la importancia de esa sección en la evaluación de la política editorial de las revistas. No realizaron, sin embargo, ningún análisis relativo a las orientaciones éticas de estas revistas.

En nuestra muestra, el número de revistas que hacen referencia a los aspectos éticos de la investigación en seres humanos en las Instrucciones a los Autores fue bajo
$(20,8 \%)$, incluso con los criterios extremamente flexibles de evaluación utilizados en este estudio.

Las revistas de medicina y enfermería presentaron mayor preocupación sobre las orientaciones éticas que las revistas no médicas, clasificadas en los grupos de misceláneas y odontología, sugiriendo que el debate ético de la experimentación en seres humanos está más difundido en las áreas médicas y de enfermería que en otras áreas de ciencias de la salud. En el área de odontología, se observa que las orientaciones éticas contenidas en el Código de Ética Odontológica, extremamente claras y precisas, son poco reflejadas en la política editorial de los periódicos.

El Código de Nüremberg y la Declaración de Helsinki son documentos que mencionan las experiencias médicas en seres humanos. La resolución del CNS-MS de 1988 hace referencia a la investigación en el área de la salud. Por otro lado, la actual legislación brasileña, resolución 196/96 del CNS-MS, alude a cualquier investigación involucrando a seres humanos, incluso en áreas como la sociología, la economía y el medio ambiente.

Brackbill y Hellegers (19) realizaron entrevistas con editores de revistas médicas y observaron que el $27 \%$ procedían a la revisión ética de los artículos enviados para publicación y $21,3 \%$ exigian que los estudios fuesen previamente aprobados por Comités Institucionales.

Rickkert et al (20), analizando artículos publicados en revistas de geriatría de lengua inglesa, notaron que en el $29 \%$ había consentimiento informado, en el $21 \%$ era mencionada la aprobación previa por el Comité Institucional y en los restantes no había referencia a los aspectos éticos. Amdur y Biddle (17) analizaron las Instrucciones a los Autores de 102 revistas científicas de biomedicina de lengua inglesa y observaron que $24 \%$ de ellas no mencionaban los aspectos éticos de la investigación en seres 
humanos; Rennie y Yank (13) realizaron un análisis informal de 53 artículos sobre consentimiento informado y aprobación por el Comité Institucional y obtuvieron resultados semejantes.

Independientemente de estos autores, enfatizaremos la necesidad de incrementar el debate, buscando ampliar y detallar las orientaciones relativas a la ética en la política editorial de las revistas científicas médicas y biomédicas. La comparación con nuestros resultados, en que solamente $20,8 \%$ de las revistas se refieren de alguna manera a la ética de la investigación en seres humanos, demuestra que las publicaciones brasileñas están poco involucradas en este debate.

Las Instrucciones a los Autores de las revistas científicas brasileñas, analizadas en el presente estudio, enfatizan aspectos técnicos de los artículos, principalmente en cuanto a la forma y a las referencias bibliográficas. Este caso es observado también en los RUMSRB (14-16) que a pesar de incluir aspectos específicamente relacionados con la ética, dedican mayor espacio y exigen más detalles respecto de la parte técnica de los manuscritos (13).

Observamos que en las seis revistas que hacen referencias a la Declaración de Helsinki, ninguna indica cómo encontrar este documento publicado en lengua portuguesa o incluso inglesa. Realizamos una investigación informal de la copia actualizada de este documento en portugués y tuvimos dificultad en obtenerla $(9,21)$, confirmándose la reducida divulgación de este texto en el medio científico y editorial brasileño.

El consentimiento del paciente para participar de un experimento científico es citado ocho veces en las 139 revistas brasileñas estudiadas, constatando que solamente en tres revistas la redacción del texto en las Instrucciones a los Autores se refiere al hecho que el consentimiento debe ser obtenido después que el paciente ha sido informado sobre el estudio que se realizará.
Hossne y Vieira (22) critican el término normalmente usado en lengua portuguesa, "consentimiento postinformado" y proponen la redacción "consentimiento esclarecido", más adecuado al principio de que los individuos deben ser no solamente informados previamente, sino que esclarecidos adecuadamente sobre el experimento a ser realizado. La actual norma que reglamenta la investigación sobre seres humanos en el Brasil, Resolución 196/96 del CNS-MS, adopta el término "consentimiento libre y esclarecido".

Los RUMSRB son citados en las Instrucciones a los Autores de 11 revistas, aunque conviene agregar que en seis casos, las referencias se limitan solamente a los aspectos técnicos, principalmente relacionadas con las citaciones bibliográficas. Solamente dos revistas publicaron los RUMRSB en portugués $(15,16)$; nueve revistas que citan este documento, mencionan a la publicación en lengua inglesa. Solamente el Periódico Brasileño de Urología es claro al afirmar que los trabajos deben ser encaminados de acuerdo con estas normas, en tanto que las otras revistas afirman que sus normas son basadas en los RUMSRB. El Periódico de Pediatria es la única revista que cita los RUMSRB y, concomitantemente, hace referencia explicita a las orientaciones éticas, exigiendo aprobación por la Comisión de Ética y respeto de la Resolución 196/96 del CNS-MS.

Estos datos indican que los RUMSRB es un documento poco divulgado y conocido en el medio científico brasileño. Por otro lado, las orientaciones éticas de los RUMSRB son consideradas extremamente frágiles (13), al solicitar a los autores que al describir experimentos con seres humanos:

indiquen si los procedimientos fueron realizados de acuerdo con los estándares éticos del comité responsable para la experimentación humana (institucional o regional) y con la Declaración de Helsinki de 1975, de acuerdo a la revisión de 1983 (15). 
Análisis de los aspectos éticos de la investigación en seres humanos contenidos en las Instrucciones a los Autores de 139 revistas científicas brasileñas - T. Sardenberg y cols.

Las tres revistas que hacen referencias genéricas a la ética en sus Instrucciones a los Autores, utilizan los términos principios, normas y estándares éticos, no estableciendo ninguna especificación sobre estas orientaciones $\mathrm{y}$, por lo tanto, constituyendo orientaciones éticas frágiles e inconsecuentes.

Las 29 revistas que poseen orientaciones respecto de los aspectos éticos, presentaron un comportamiento semejante a los observados por Amdur y Biddle (17) en relación a la manera como las Instrucciones a los Autores solicitan a los investigadores que informen sobre los aspectos éticos de los estudios enviados para publicación. La mayoría $(57,8 \%)$ pide que las informaciones sean incluidas en el texto del artículo, generalmente en la parte de material y métodos. Por otro lado, el número de revistas que no tiene ninguna referencia sobre este aspecto puede ser considerado elevado $(34,4 \%)$ sugiriendo que estas publicaciones pueden no ejercer un control adecuado sobre sus propios criterios para la publicación de artículos científicos.

La importancia de la publicación de los resultados de las investigaciones realizadas es, actualmente, incuestionable, tanto del punto de vista de la divulgación del conocimiento científico, como en relación a los principios de la ética (23). La publicación de artículos científicos es uno de los principales criterios de evaluación de la productividad de los investigadores y de las Instituciones de investigación. Por lo tanto, la política editorial de las revistas científicas reviste una gran importancia en el desenvolvimiento científico.

La integridad del sistema científico de investigación depende de la seriedad de los investigadores (17). Entretanto, la política editorial de las revistas científicas, intentando mejorar no solamente los aspectos técnicos de los artículos, sino también la ética de los estudios, podría contribuir de modo efectivo al mejoramiento global de las publicaciones brasileñas de medicina, biomedicina, odontología, enfermería y ciencias generales.

Tabla I - Frecuencias absoluta y relativa de las referencias éticas de la investigación en seres humanos contenidas en las Instrucciones a los Autores de revistas científicas brasileñas.

Comité o Comisión de Ética

Declaración de Helsinki o Código de Nüremberg

Consentimiento Esclarecido

Requisitos Uniformes

Principios / Normas / Estándares Éticos

Sin Referencias Ética

$\mathrm{n}=$
$17(12,2 \%)$

$3(2,1 \%)$

$1(0,7 \%)$

$5(3,5 \%)$

$3(2,1 \%)$

$110(79,1 \%)$

139 (100\%) 
Tabla II - Frecuencia de referencias éticas de la investigación en seres humanos contenidas en las Instrucciones a los Autores de revistas científicas brasileñas, clasificadas de acuerdo con las especialidades.

Cirugía (16)

Clínica Médica (14)

8

\section{8}

Pediatría (4)

5

2

2

Psiquiatría (4)

0

Medicina General (26)

0

7

19

Medicina Social/Ocupacional (7)

1

Oncología (3)

0

3

Especialidades (5)

14

Enfermería (Practicantes) (5)

2

Miscelánea (11)

0

Odontología (44)

3

Frecuencia Absoluta

29

110

Frecuencia Relativa

$(20,8 \%)$

$(79,1 \%)$

$\mathrm{n}=139$ 
Análisis de los aspectos éticos de la investigación en seres humanos contenidos en las Instrucciones a los Autores de 139 revistas científicas brasileñas - T. Sardenberg y cols.

Tabla III- Frecuencia de los tipos de referencias éticas de la investigación en seres humanos contenidos en las Instrucciones a los Autores de revistas científicas brasileñas clasificadas de acuerdo con las especialidades.

\section{Orientaciones Éticas}

\begin{tabular}{|c|c|c|c|c|c|c|c|}
\hline \multicolumn{2}{|l|}{$\begin{array}{l}\text { Especialidades } \\
\text { (nºde revista) }\end{array}$} & $\begin{array}{l}\text { Comisión } \\
\text { de Ética }\end{array}$ & $\begin{array}{l}\text { D.Helsinki/ } \\
\text { Nüremberg }\end{array}$ & $\begin{array}{l}\text { Consent } \\
\text { Esclar. }\end{array}$ & $\begin{array}{l}\text { Princ/Normas } \\
\text { Estándares }\end{array}$ & $\begin{array}{l}\text { Requisitos } \\
\text { Uniformes }\end{array}$ & $\begin{array}{l}\text { Sin ref } \\
\text { Éticas }\end{array}$ \\
\hline \multicolumn{2}{|l|}{ Cirugía (16) } & 5 & 1 & 0 & 0 & 2 & 8 \\
\hline \multicolumn{2}{|c|}{ Clínica médica (14) } & 2 & 0 & 1 & 1 & 1 & 9 \\
\hline \multicolumn{2}{|l|}{ pediatría (4) } & 1 & 1 & 0 & 0 & 0 & 2 \\
\hline \multicolumn{2}{|l|}{ Psiquiatría (4) } & 0 & 0 & 0 & 0 & 0 & 4 \\
\hline \multicolumn{2}{|c|}{ Medicina general (26) } & 5 & 1 & 0 & 0 & 1 & 19 \\
\hline \multicolumn{2}{|c|}{ Medicina social / } & 0 & 0 & 0 & 0 & 1 & 6 \\
\hline \multicolumn{8}{|l|}{ Ocupacional (7) } \\
\hline \multicolumn{2}{|l|}{ Oncología (3) } & 0 & 0 & 0 & 0 & 0 & 3 \\
\hline \multicolumn{2}{|l|}{ Especialidad (5) } & 1 & 0 & 0 & 0 & 0 & 4 \\
\hline \multicolumn{2}{|l|}{ Enfermería (5) } & 1 & 0 & 0 & 1 & 0 & 3 \\
\hline \multicolumn{2}{|l|}{ Miscelánea (11) } & 0 & 0 & 0 & 0 & 0 & 11 \\
\hline \multicolumn{2}{|l|}{ Odontología (44) } & 2 & 0 & 0 & 1 & 0 & 41 \\
\hline \multicolumn{2}{|l|}{$\mathrm{n}=139(100 \%)$} & $17(12,2 \%)$ & $3(2,1 \%)$ & $1(0,7 \%)$ & $3(2,1 \%)$ & $5(3,5 \%)$ & $110(79,1 \%)$ \\
\hline D. Helsinki & $=$ & \multicolumn{6}{|c|}{ Declaración de Helsinki } \\
\hline Nüremberg & $=$ & \multicolumn{6}{|c|}{ Código de Nüremberg } \\
\hline Consent. Esclar. & $=$ & \multicolumn{6}{|c|}{ Consentimiento Esclarecido } \\
\hline Princi. & $=$ & \multicolumn{6}{|c|}{ Principios } \\
\hline Unifor. & $=$ & \multicolumn{6}{|l|}{ Uniformes } \\
\hline Ref. & $=$ & \multicolumn{6}{|l|}{ Referencias } \\
\hline
\end{tabular}

Tabla IV - Frecuencia de la manera como las Instrucciones a los Autores de revistas científicas solicitan que las informaciones referentes a los aspectos éticos de las investigaciones en seres humanos sean proporcionadas.

Incluir en el texto del artículo

Carta firmada por los autores

Copia de la autorización de la Comisión de Ética

Subentendido ( el autor asume la responsabilidad)

Sin referencias

$\mathrm{n}=$
$15 \quad(51,7 \%)$

$2 \quad(6,8 \%)$

$1 \quad(3,4 \%)$

$1 \quad(3,4 \%)$

$10 \quad(34,4 \%)$

$29 \quad(100.0 \%)$ 
Cuadro 1. Revistas científicas que hacen referencia a la Declaración de Helsinki, aisladamente o en conjunto con otras orientaciones, en las Instrucciones a los Autores.

-Revista Colégio Brasileiro de Cirurgiões

-Revista Brasileira de Oftalmologia

-Revista do Hospital de Clínicas de Porto Alegre

-Revista Brasileira de Medicina

-Revista Brasileira de Medicina - Ginecologia e

Obstetrícia

-Revista Paulista de Pediatria
Cuadro 2. Revistas científicas que hacen referencia al consentimiento del paciente, aisladamente o en conjunto con otras orientaciones, en las Instrucciones a los Autores.

- Arquivo Brasileiro de Cardiologia

- Jornal Brasileiro de Nefrologia

- Revista Brasileira de Medicina

- Revista Brasileira de Medicina-Ginecologia e Obstetrícia

- Revista Brasileira de Anestesiologia

- Revista Latino Americana de Enfermagem

- Revista da Pós-Graduação

- Revista de Odontologia da USP

Cuadro 3 - 139 revistas brasileñas clasificadas de acuerdo con las referencias a los aspectos éticos de la investigación en seres humanos en las Instrucciones a los Autores.

\section{Comisión o Comité de Ética}

Jornal de Pneumologia

Revista de Medicina da PUCRS

Revista Colégio Brasileiro de Cirurgiões

Revista Brasileira de Ginecologia e Obstetrícia

Revista Brasileira de Otorrinolaringologia

Revista Paulista de Medicina

Jornal Brasileiro de Nefrologia

Revista Brasileira de Medicina

Revista Brasileira de Medicina - Ginecologia e Obstetrícia

Revista Brasileira de Anestesiologia

Jornal de Pediatria

Braziliam Journal of Medical and Biological

Research

Revista Saúde (UF Sta Maria)

Revista Latino Americana de Enfermagem

Acta Cirúrgica Brasileira

Revista da Pós-Graduação

Revista de Odontologia

Declaración de Helsinki/Código de Nüremberg

Revista Brasileira de Oftalmologia

Revista Paulista de Pediatria

Revista do Hospital de Clínicas de Porto Alegre

Consentimiento esclarecido

Arquivos Brasileiro de Cardiologia
Principios / Normas / Estándares Éticos

Revista Brasileira de Reumatologia

Revista Brasileira de Enfermagem

RFO - UPF

Requisitos Uniformes

Jornal Brasileiro de Urologia

Revista de Saúde Pública

Revista Médica de Minas Gerais

Revista Brasileira de Crescimento e

Desenvolvimento Humano

Revista Brasileira de Cirurgia Cardiovascular

\section{Sin referencias éticas}

Revista da Sociedade Brasileira de Cirurgia Plástica

Revista Brasileira de Ortopedia

Acta Ortopedica Brasileira

Jornal Brasileiro de Ginecologia

Revista Brasileira de Colo-Proctologia

Revista Brasileira de Cirurgia

Revista Brasileira de Mastologia

Revista de Ginecologia \& Obstetrícia

Revista Brasileira e Latino Americana de Marca

Passo e Arritmia

The Brazilian Journal of Infectious Diseases

Revista da Sociedade de Cardiologia do Estado de

São Paulo

Revista Brasileira de Clínica \& Terapêutica 
Análisis de los aspectos éticos de la investigación en seres humanos contenidos en las Instrucciones a los Autores de 139 revistas científicas brasileñas - T. Sardenberg y cols.

\begin{tabular}{|c|c|}
\hline Revista Brasileira de Medicina Psicossomática & Anais da Acadêmia Brasileira de Ciências \\
\hline Revista da Sociedade Brasileira de Medicina Tropical & Revista Brasileira de Biologia \\
\hline Revista do Instituto de Medicina Tropical de São & Journal of the Brazilian Society of Microbiology \\
\hline Paulo & Revista Brasileira de Educação Médica \\
\hline Revista HiperAtivo & Anais Brasileiros de Dermatolgia \\
\hline Revista de Homeopatia & Arquivos de Neuro-Psiquiatria \\
\hline Pediatria (São Paulo) & Revista Brasileira de Neurologia \\
\hline Arquivos Brasileiros de Pediatria & Arquivos de Gastroenterolgia \\
\hline Revista da Escola de Enfermagem da USP & Revista Paulista de Odontologia \\
\hline Revista Paulista de Enfermagem & Odontologia Gaucha \\
\hline Revista Gaucha de Enfermagem & Odontologia USF \\
\hline Revista de Psiquiatria Clínica & Revista de Odontopediatria - Atualização e Clínica \\
\hline Psiquiatria Biológica & Revista de Odontologia da UNICID \\
\hline Revista ABP - APAL & Revista de Odontologia da UNESP \\
\hline Jornal Brasileira de Psiquiatria & Revista da Faculdade de Odontologia da UF de \\
\hline Revista da Associação Médica Brasileira & Pelotas \\
\hline Ars Cvrandi & Revista da Faculdade de Odontologia da UF da Bahia \\
\hline A Folha Médica & Revista de Periodontia da Sobrape \\
\hline Revista de Medicina & Revista Brasileira de Odontologia \\
\hline Arquivos Médicos dos Hospitais e da Faculdade de & Revista Científica CENBIOS \\
\hline Ciências Médicas da Sta Casa de São Paulo & Revista do Instituto Brasileiro de Implantologia \\
\hline Revista de Medicina do Hospital Universitário & Revista do CROMG \\
\hline Revista Médica do IAMSPE & Revista Brasileira da Ciência Estomalógicas \\
\hline Iatros & Revista de Odontologia da Faculdade de Odontologia \\
\hline Salusvite & de Bauru \\
\hline Revista de Saúde do Distrito Federal & Revista da Faculdade de Odontologia de Lins \\
\hline Revista do Hospital das Clínicas FMUSP & Revista da Faculdade de Odontologia da UFRG \\
\hline Arquivos Brasileiros de Medicina & Arquivos do Centro de Estudos do Curso de \\
\hline Anais Paulista de Medicina e Cirurgia & Odontologia \\
\hline Arquivos Catarinense de Medicina & Stomatos \\
\hline Revista de Ciências PUCAMP & Revista de Odontologia do Brasil Central \\
\hline Medicina & Revista SPRO \\
\hline Anais da Academia Nacional de Medicina & Revista Goiânia de Ortodontia \\
\hline Jornal Brasileiro de Medicina & Revista do Centro de Estudos da FO UERJ \\
\hline Revista Goiana de Medicina & Revista Dental Press de Ortodontia e Ortopedia \\
\hline Revista da Faculdade de Ciências Médicas da & Maxilar \\
\hline Unicamp & Revista Odontologia de Santo Amaro \\
\hline Mundo da Saúde & Brazilian Endodontia Journal \\
\hline Revista do Instituto Adolfo Lutz & Dens \\
\hline Cadernos de Saúde Pública & Jornal Brasileiro de Odontopediatria e Odontologia \\
\hline Saúde em Debate & do Bebê \\
\hline Revista Brasileira de Saúde Ocupacional & Implantares \\
\hline História - Ciência - Saúde - Manguinhos & FOPLAC em Revista \\
\hline Revista Brasileira de Cancerologia & Odontologia - Ensino e Pesquisa \\
\hline Acta Oncológica Brasileira & Odontologia Clínica \\
\hline Oncologia Atual & Odonto Pope \\
\hline Jornal Brasileiro de Patologia & Odonto \\
\hline Revista de Ciências Biomédicas & Odonto 2000 \\
\hline Revista de Ciências Farmacêuticas & Brazilian Dental Journal \\
\hline Revista de Farmácia e Bioquímica da USP & Revista APCD \\
\hline Revista de Nutrição da PUCAMP & Revista ABO - Nacional \\
\hline Revista Brasileira de Análises Clínicas & Revista Ortodontia \\
\hline Brazilian Journal of Genetics & Revista do Instituto da Saúde \\
\hline
\end{tabular}




\section{Referencias}

1. The Nuremberg Code (1947). BMJ 1996;313:1448.

2. World Medical Association. Declaration of Helsinki. JAMA 1997;277:925-6.

3. Conselho Federal de Medicina. Código de Ética Médica: Resoluçâo 1246;1988.

4. Conselho Federal de Enfermagem. Código de Ética dos Profissionais de Enfermagem: Resoluçâo 160; 1993.

5. Conselho Federal de Odontologia. Código de Ética Odontológica: Resoluçâo 179; 1992.

6. Conselho Nacional da Saúde. Ministério da Saúde. Normas de Pesquisa em Saúde: Resolução 01; 1988.

7. Conselho Nacional da Saúde. Ministério da Saúde. Diretrizes e Normas Regulamentadoras de Pesquisa Envolvendo Seres Humanos: Resolução 196; 1996.

8. Décourt LV. Ética da investigação clínica. En: Gonçalves EL, (ed). Pesquisa médica. São Paulo: E.P.U. - CNPQ; 1983. p.11-23.

9. Vieira S, Hossne, WS. A ética em pesquisa. En: Experimentação com seres humanos. São Paulo: Editora Moderna;1987. p. 51-70.

10. Smith R. Informed consent: the intricacies. BMJ 1997; 314:1059-60.

11. Doyal L. Informed consent in medical research (journals should not publish research to which patients have not given fully informed consent) with three exceptions. BMJ 1997; 314: 1107-11.

12. Tobias JS. BMJ's present policy (sometimes approving research in which patients have not given fully informed consent) is wholly correct. BMJ 1997; 314:111-4.

13. Rennie D, Yank V. Disclosure to the reader of institutional review board approval and informed consent. JAMA 1997; 277: 922-3.

14. International Committee of Medical Journal Editors. Uniform Requeriments for Manuscripts Submitted to Biomedical Journals. JAMA 1997; 277:927-34.

15. Comitê Internacional de Editores de Revistas Médicas. Requisitos Uniformes para Originais Submetidos a Revistas Biomédicas. Journal of Pediatrics 1997; 73:213-24.

16. Comitê Internacional de Editores de Revistas Médicas. Critérios Uniformes para Encaminhamento de Artigos para Publicação em Jornais de Área Biomédica. Journal Brasileiro de Urologia 1992; 18:65-70.

17. Amdur RJ, Biddle C. Institutional Review Board approval and publication of human research results. JAMA 1997; 277: 909-14.

18. Goldenberg S, Población DA, Gomes PO. Editoração de revistas científicas. Análise das instruções aos autores de 19 revistas brasileiras. Acta Cirurgica Brasileira 1995; 10: 55-60.

19. Brackbill Y, Hellengers AE. Ethics and editors. Hastings Center Report 1980;10:20-4.

20. Rikkert MGMO, Have HAMJ, Hoefnagels WHL. Informed consent in biomedical studies on aging: survey of four journals. BMJ 1996; 313:1117.

21. Conselho Federal de Medicina. Declaração de Helsinque adotada pela $18^{\mathrm{a}}$ Assembléia Mundial de Médicos, Helsinque: Resolução 1098; 1983.

22. Hossne WS, Vieira S. Experimentação com seres humanos: aspectos éticos. En: Segre M, Cohen C, (eds). Bioética. São Paulo: EDUSP; 1995. p. 127-46.

23. Pearn J. Publication: an ethical imperative. BMJ 1995; 310: 1313-5. 